\title{
Fermi LAT and GBM collaboration results on GRB
} 200415A

N. Di Lalla ${ }^{a, *}$, A. Beretta ${ }^{b}$, N. Omodei ${ }^{a}$, F. Piron $^{c}$, S. Razzaque ${ }^{d}$, O. J. Roberts ${ }^{e}$, E. Bissaldi $^{f, g}$, P. Veres $^{h}$, M. Baring ${ }^{i}$, on behalf of the Fermi-LAT Collaboration and Fermi-GBM Team

${ }^{a}$ W. W. Hansen Experimental Physics Laboratory, Kavli Institute for Particle Astrophysics and Cosmology, Department of Physics and SLAC National Accelerator Laboratory, Stanford University

${ }^{b}$ Dipartimento di Fisica, Università degli Studi di Perugia, Perugia, Italy

${ }^{c}$ Laboratoire Univers et Particules de Montpellier, Université Montpellier, CNRS/IN2P3, Montpellier, France

${ }^{d}$ Centre for Astro-Particle Physics (CAPP) and Department of Physics, University of Johannesburg, Johannesburg, South Africa

e Universities Space and Research Association, Huntsville, AL, USA

${ }^{f}$ Dipartimento di Fisica, “M. Merlin” dell'Università e del Politecnico di Bari, Bari, Italy

${ }^{g}$ Istituto Nazionale di Fisica Nucleare, Bari, Italy

${ }^{h}$ Center for Space Plasma and Aeronomic Research, University of Alabama in Huntsville, Huntsville, AL, USA

${ }^{i}$ Department of Physics and Astronomy, Rice University, Houston, TX, USA

E-mail: niccolo.dilalla@stanford.edu

\footnotetext{
${ }^{*}$ Presenter
} 
Magnetars are neutron stars with the strongest magnetic fields known in the Universe, with an intensity up to a thousand times higher than typical neutron stars. Rarely, magnetars can produce enormous eruptions, called Magnetar Giant Flares (MGFs), consisting of short-duration bursts of hard X-rays and soft gamma rays - a bright and variable initial spike lasting a few tenths of a second and a significantly dimmer pulsating tail lasting a few hundreds of seconds that can only be detected from MGFs within our galaxy. On April 15, 2020, a short bright burst of MeV gamma rays triggered the Gamma-Ray Burst Monitor (GBM) aboard the Fermi spacecraft, called GRB 200415A and localized by the InterPlanetary Network (IPN) inside the disk of the nearby Sculptor galaxy. 19 seconds later, and for nearly 300 seconds, the Large Area Telescope (LAT) detected $\mathrm{GeV}$ photons in spatial coincidence with the signal at lower energies. In this talk we present the recently published results of the GBM and LAT analysis on GRB 200415A. Our detailed analysis shows that the low-energy emission has very peculiar properties typically observed in flares from nearby magnetars, while the GeV detection is consistent with the IPN localization and spatially associated with the Sculptor galaxy. Hence, we infer that gamma rays likely originated from the MGF in Sculptor, and not from a cosmological gamma-ray burst, and we suggest that the GeV signal is generated by an ultra-relativistic outflow that first radiates the prompt MeV-band photons. This discovery represents the first detection of the high-energy emission from an MGF and proves that extragalactic MGFs may indeed disguise as short GRBs and constitute a small fraction of current short GRB samples.

$37^{\text {th }}$ International Cosmic Ray Conference (ICRC 2021)

July 12th - 23rd, 2021

Online - Berlin, Germany 


\section{Introduction}

Magnetars are neutron stars with the strongest magnetic fields known in the Universe, with intensities up to a thousand times higher than typical neutron stars [1]. They feature rotation periods ranging from fractions of a second up to several seconds, and a luminosity in X-rays of the order of $10^{31-36} \mathrm{erg} / \mathrm{s}$. Beside the steady emission, magnetars can manifest different types of flaring activities. First, they emit recurrent, short duration bursts with period of about $0.1 \mathrm{~s}$ and, for this reason, magnetars are also known with the name of Soft Gamma-Repeaters. Second, they can experience long-lasting active phases of increased luminosity lasting years. Finally, the most extreme and rare form of emission of magnetars are giant flares.

Magnetar Giant Flares (MGFs) are enormous eruptions consisting of short-duration bursts of hard X-rays and soft gamma-rays with luminosity of about $10^{44-47} \mathrm{erg} / \mathrm{s}$ [2, 3]. The initial bright spike usually lasts just a fraction of a second and it is followed by a significant dimmer soft $\mathrm{X}$-ray tail lasting hundreds of seconds modulated with the spin of the magnetar. Giant flares are poorly understood but they are believed to result from extreme star-quakes induced by a sudden reconfiguration of the enormous magnetic field. This probably causes fractures of the neutron star crust and the consequent release of hot plasma into the star magnetosphere.

Just a handful of MGFs has been observed over the past 50 years: two coming from the Milky Way galaxy, one from the Large Magellanic Cloud and four others from nearby galaxies, namely M31, M81, M83 and NGC 253 [4-8]. Interestingly, the 2020-event from NGC 253 was actually the first giant flare known to occur since Fermi's launch in 2008 and the first observed up to $\mathrm{GeV}$ energies. When a giant flare occurs within our Galaxy, the initial flash can be so intense that it can easily saturate all the observing telescopes, while the dimmer pulsating tail may remain detectable. On the other hand, when the magnetar is located in a nearby galaxy, tens of millions of light-years away, the periodic tail becomes very hard to observe and the event appears essentially as a short bright pulse lasting hundreds of milliseconds that totally resembles a short gamma-ray burst (GRB). Because of their difference in intrinsic luminosity, short GRBs are visible at the Gpc scale, while MGFs can be detected only up to 10-15 Mpc. The fact that the closest short GRB known to date, associated with GW 170817, has a measured distance of about $40 \mathrm{Mpc}$ indicates that MGFs are probably much more common events in the Universe than short GRBs [9].

\section{Observations of GRB 200415A}

On April 15th, 2020 at 08:45:05 UTC, the Gamma-ray Burst Monitor (GBM) on-board Fermi was triggered by what first seemed to be an extremely bright short GRB [12]. The same event was also observed by other space instruments, Swift-BAT, Konus-Wind, Mars-Odyssey (HEND) and INTEGRAL (SPI-ACS), all part of the so-called InterPlanetary Network or IPN [13]. Orbiting at different positions around the Earth and Mars, they can together triangulate GRB positions with great precision using differences in photon arrival times. In the case of GRB 200415A, the GRB was localized by the IPN network into a $17 \mathrm{arcmin}^{2}$ region spatially consistent with the central part of NGC 253, known as the Sculptor galaxy (Figure 1, left panel). NGC 253 is an active star-bursting galaxy, located at about 3.5 Mpc from Earth [14]. The probability to obtain a chance coincidence between the GRB and the Sculptor galaxy was estimated to be as small as 1 in 230 thousands [9]. 

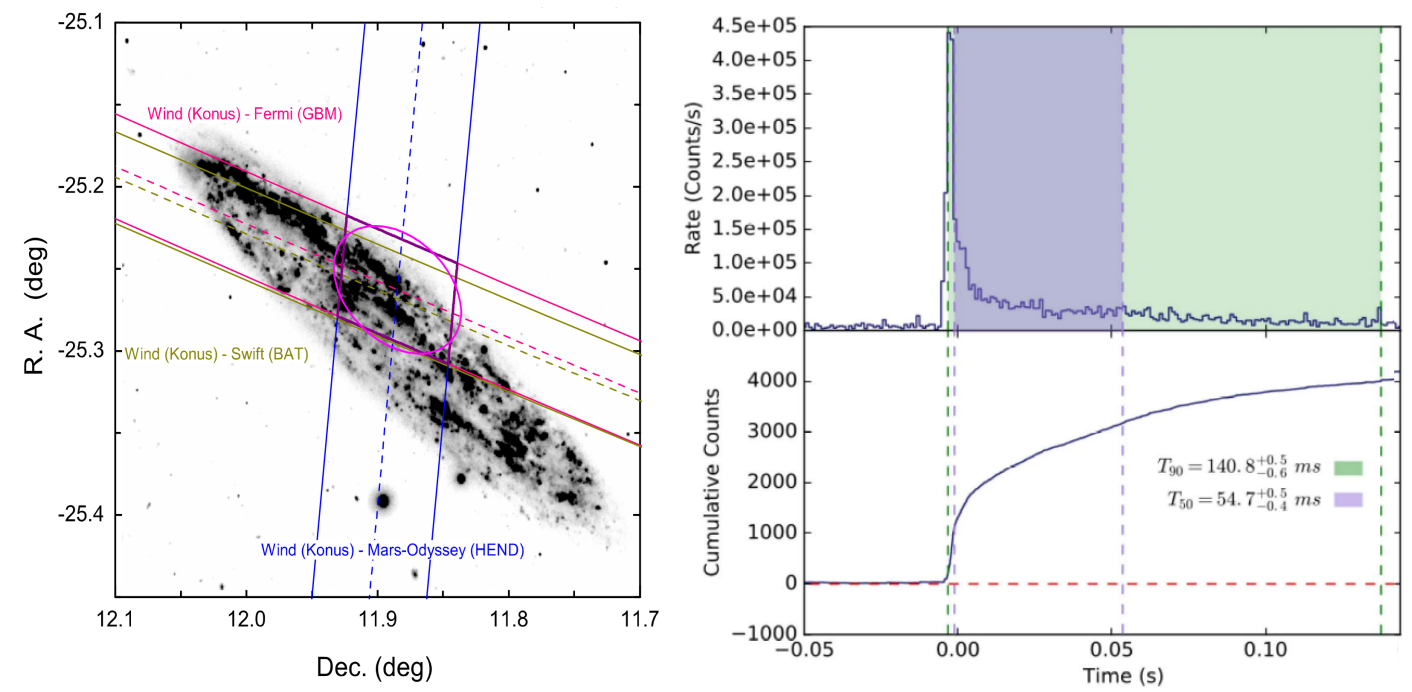

Figure 1: Left: The IPN localization superimposed on the image of the NGC 253 galaxy together with the Swift-BAT, Konus-Wind, Mars Odyssey-HEND relative annuli. Image adapted from [13]. Right: Lightcurve of GRB 200415A using the Swift-BAT data, with T90 and T50 durations shown in green and purple. Image adapted from [12].

At the time of the detection, only the KAGRA interferometer [10] was observing but no emission in GW was detected despite the short distance. A VLA [11] campaign in radio, 4.3-51.2 days after the event trigger, resulted in no new counterpart [12].

\section{Fermi-GBM analysis}

After a careful analysis, it was discovered that for a few ms the flux of the GRB exceeded the bandwidth limit between GBM and Fermi spacecraft, leading to a significant loss of photons coming from the highest peak [12]. To circumvent this issue, for timing analysis and correction of the saturated peak, data from Swift-BAT were also used and analyzed using a pipeline called GUANO, that was initially developed for non-triggered GRBs [15]. The burst was entirely outside the BAT field of view but was sufficiently bright to penetrate the side shield and and to generate a substantial signal in the detector. From the BAT light-curve the measured T90 is $141 \mathrm{~ms}$ (Figure 1, right panel), well within the range for short GRBs. To distinguish between a cosmological GRB and a flare from a nearby magnetar, it was fundamental to look at the spectral properties of this event, summarized in Figure 2. Panels (a), (b) and (c) are the light-curves with $0.2 \mathrm{~ms}$ of resolution of a Fermi-GBM BGO detector (a), a Fermi-GBM NaI detector (b) and Swift-BAT (c). The saturation of the GBM detectors is indicated by an horizontal black line covering the last part of the second time interval (shown in orange) used for spectral analysis. The bright peak observed by the BAT during these $\sim 2 \mathrm{~ms}$ is almost missing in the GBM light curves. Since the saturation affects all detectors and all energies similarly, the spectral shape could be fitted while the overall flux was underestimated. The flux was then normalized using BAT data in the same energy range and time interval. Panel (d), on the right, shows the comptonized spectra for four time intervals, while panel (e) shows the enlargement with a $12-\mu$ s resolution of the first peak colored in red. 

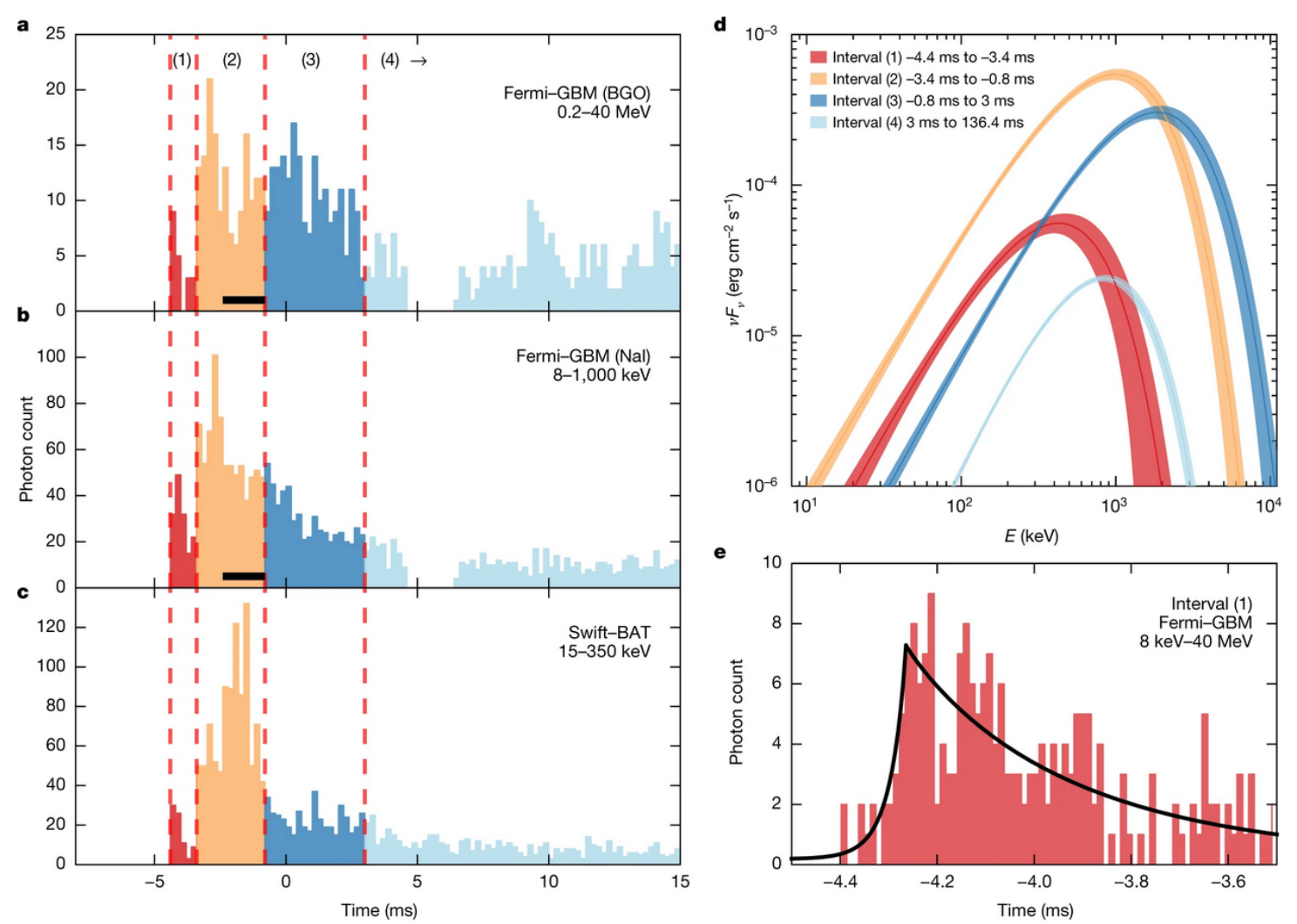

Figure 2: Panels (a), (b) and (c) are respectively the light-curves of a Fermi-GBM BGO detector, a Fermi-GBM NaI detector and Swift-BAT with $0.2 \mathrm{~ms}$ of resolution. The red dashed vertical lines and color indicate the four time intervals used for the analysis. The loss of flux due to saturation was $47.3 \%$ in the part of the orange interval marked with an horizontal black line. Panel (d) shows the best-fit comptonized spectra for the corresponding time intervals. Panel (e) is the enlargement of the first pulse in the red time interval with a temporal resolution of $12 \mu$ s. Image taken from [12].

The GBM analysis resulted in a series of unusual characteristics for a short GRB, suggesting instead a magnetar origin [16]. First of all, the rise time was less than $100 \mu \mathrm{s}$, which is extremely unlikely for a GRB but distinctive of the onset of a giant flare. Then a substantial sub-millisecond spectral variability, a photon index ( $\alpha$ ) relatively constant at $\alpha \approx 0$ (highly unusual for a short GRB) and finally a weak evidence of quasi-periodic oscillations at $180 \mathrm{~Hz}$ in the burst decay $(2.5 \sigma)$, possible signature of the seismic vibrations of the star. The burst was very bright, with a highest photon energy of a few $\mathrm{MeV}$ and calculated isotropic energy and luminosity respectively equal to $E_{\text {iso }}=1.5 \times 10^{46} \mathrm{erg}$ and $L_{\text {iso }}=1.1 \times 10^{47} \mathrm{erg} / \mathrm{s}$. Unfortunately, no clear pulsation was found in the rotational frequency range $0.02-50 \mathrm{~Hz}$, as the period-modulated tail was most likely below the detection threshold for GBM given the distance of NGC 253. Nevertheless, considering that NGC 253 is an active star-forming galaxy, that favors the presence of stellar remnants like magnetars, and that the properties of this event resembled those of previously proposed extra-galactic giant-flare candidates [17], the magnetar origin is favored against the interpretation as a cosmological short GRB. 

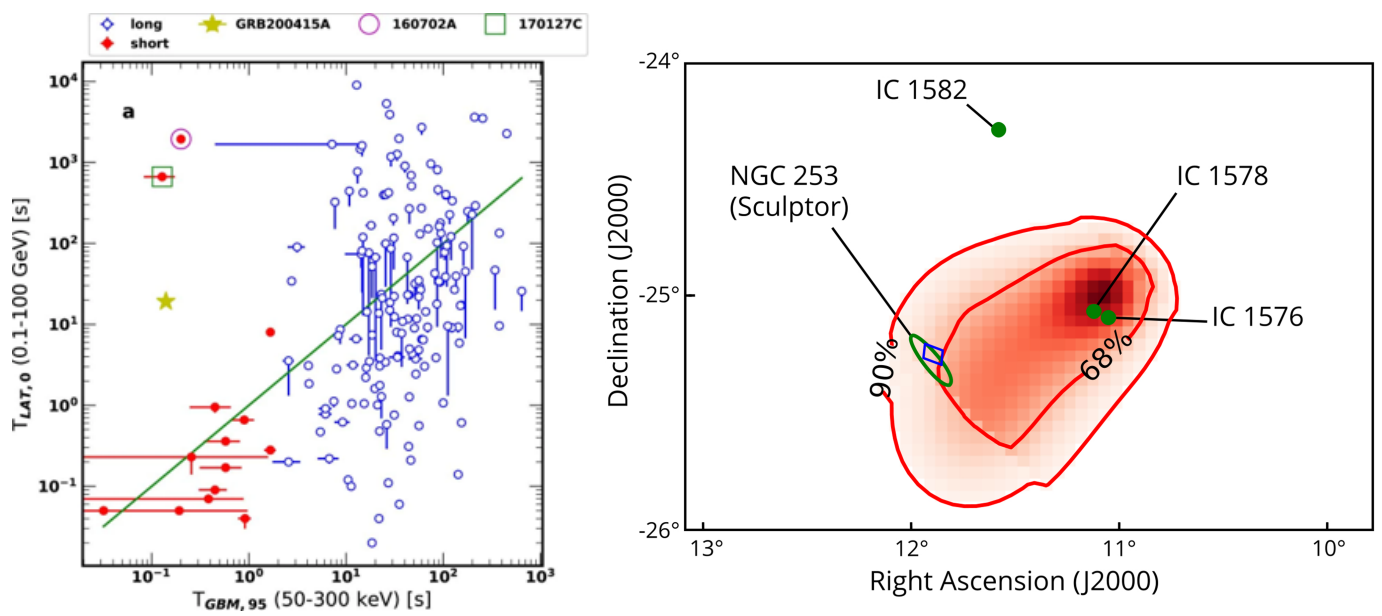

Figure 3: Left: Time of arrival of the first photon in the LAT as a function of the T95 measured by the GBM for all the GRBs detected by the LAT in the first 10 years and reported in the 2FLGC catalog [18]. Blue points are the long GRBs, while red points represent the short GRBs. GRB 200415A is added and marked with a yellow star for comparison. Right: Localization probability map computed using LAT data superimposed with the position (and extension for NGC 253) of the four galaxies from the NGC 2000 catalog in the LAT ROI. Both images adapted from [19].

\section{Fermi-LAT analysis}

The Fermi Large Area Telescope (LAT) was also observing the Sculptor region at the time of the GBM trigger and the location of the GRB remained well within the field of view up to around $500 \mathrm{~s}$ past the prompt emission. There was no detection by the LAT during the prompt phase-not uncommon since the LAT detects only a fraction of short GRBs seen by GBM-but, unexpectedly, after $19 \mathrm{~s}$ the LAT observed the first high-energy photon (slightly less than $500 \mathrm{MeV}$ ), followed by a $1.3 \mathrm{GeV}$ and a $1.7 \mathrm{GeV}$ photons after almost 5 minutes [19].

This behavior was really peculiar and never happened before in the LAT history. Figure 3, left panel, collects all the short and long GRBs detected by LAT during the first 10 years of operation [18], showing the arrival time of the first LAT photon as a function of the GBM T95, that marks the end of the sub-MeV prompt emission. This plot clearly shows that for short GRBs (in red) the LAT emission always starts before or right after the GBM emission is over in all cases except three: the yellow star is GRB 200415A, the green square is $170127 \mathrm{C}$ which was outside the LAT field of view at the trigger time and was observed later thanks to a re-pointing issued by the GBM, and the purple circle is 160702A during which Fermi was initially inside the SAA and the LAT detected the high-energy emission only once outside. In the end, GRB 200415A was actually the only short GRB within the field of view since the beginning but detected only at a later time.

In order to associate the LAT signal with the GRB 200415A detected by GBM, the first step of the analysis was to obtain a localization map for the event. This was done by running a maximum likelihood analysis and computing the map of Test Statistic (TS), which tests the presence of a new source at different positions. The maximum TS $\approx 29$ was found at $\mathrm{R} . \mathrm{A}=11.13^{\circ}, \mathrm{DEC}=-24.97^{\circ}$, and corresponds to a $\sim 5 \sigma$ detection. The TS map can then be transformed into the localization probability map, shown on the right panel of Figure 3, with NGC 253 lying at the $72 \%$ contour 


\begin{tabular}{c|c|c|c|c} 
Galaxy & NGC 1576 & NGC 1578 & NGC 1582 & NGC 253 \\
\hline LR & 2.1 & 2.9 & 0.3 & 60
\end{tabular}

Table 1: Likelihood Ratio values obtained for the four NGC 2000 galaxies in the LAT ROI. With a much larger LR value, NGC 253 is the most likely host galaxy of the source detected by LAT. Data taken from [19].

level. Beside the Sculptor galaxy in the $3^{\circ} \times 3^{\circ}$ LAT region of interest (ROI) there were three other galaxies from the NGC 2000 catalog [20] plus many fainter galaxies. The Likelihood Ratio method [21] is a standard association method applied in several studies for counterpart searches in different catalogs and was used to associate the LAT emission with one of these four galaxies. Thanks to its greater luminosity, the Sculptor galaxy is the most likely host galaxy of the source detected by the LAT with a much larger LR value with respect to the other galaxies (Table 1).

To evaluate the statistical significance of this spatial association, the probability distribution of LR values was built using a MC simulation, repeating the same analyses over a sample of $10^{5}$ random locations in the sky of the LAT excess. The plots in Figure 4 represent the cumulative distributions of the LR values obtained in the random sampling, while the vertical lines are the actual values obtained for NGC 253. Analyses shown on panel (a) calculate the LR by using the standard Rayleigh formula, while those in panel (b) make use of the actual TS map to compute the probability. The color of the distribution distinguishes between the analysis where the galaxy extension is taken (blue) or not taken (red) into account. Depending on the particular analysis considered, the resulted p-values are of the order of $10^{-3}-10^{-4}$, which translate to a False Alarm Rate ranging from 1 chance coincidence in 200 to 1800 years.

After establishing a significant spatial association with NGC 253, it is important to also associate temporally the LAT signal with the GRB detected by the GBM and to evaluate the significance of detecting three high-energy photons in a time-interval of less than 300 seconds from this region far

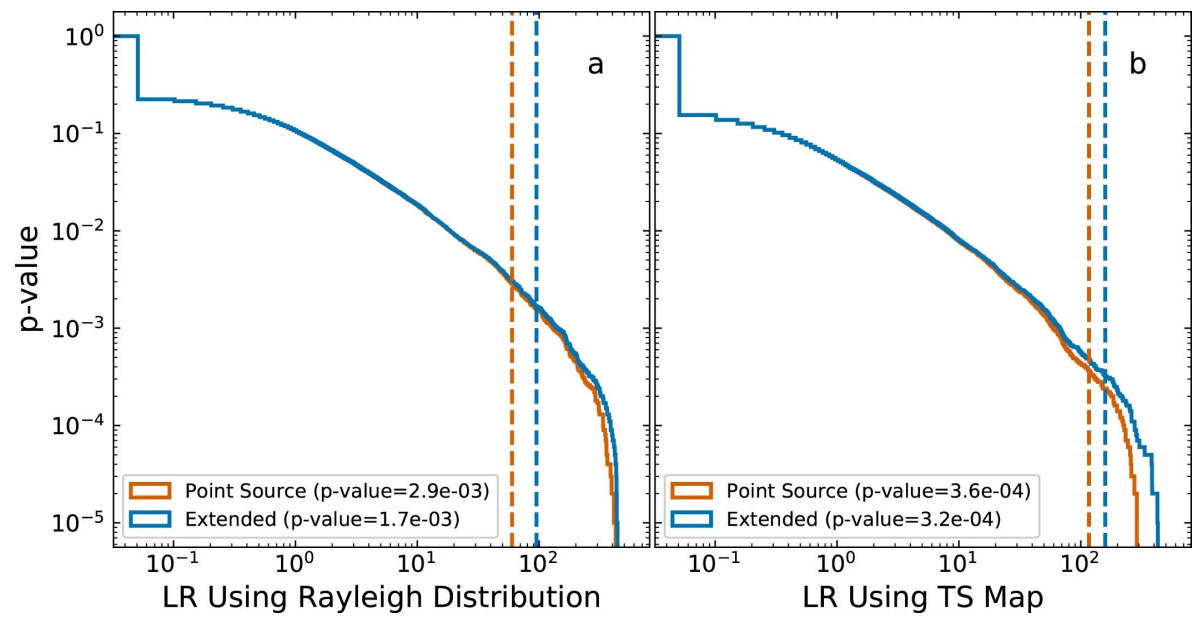

Figure 4: Distributions of the LR values obtained for the $10^{5}$ simulated ROI using the standard Rayleigh formula (panel (a) on the left) or the actual TS map (panel (b) on the right). The two colors of the distributions indicate whether the extension of the host galaxy was taken (blue) or not taken (red) into account to compute the probability. The vertical dashed lines represent the LR values obtained for NGC 253. Image taken from [19]. 


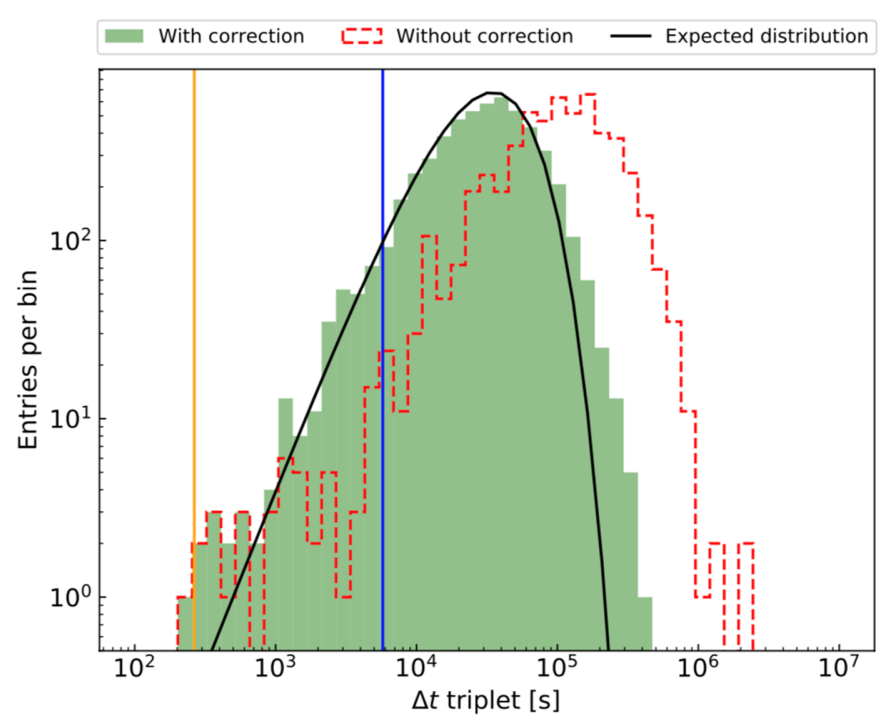

Figure 5: Distributions of the measured time intervals $\Delta t_{i}$ for each cluster of three consecutive events detected by the LAT in 12 years within a radius of 1 degree from the center of the Sculptor galaxy. The green distribution comes from the red one with the correction for the Bad Time Intervals. The expected distribution using the Poisson statistics for independent events is shown in solid black. The orange vertical line corresponds to the $\Delta t$ observed for GRB 200415A (265 s), while the vertical blue line shows the period of the Fermi orbit (5790 s). Image adapted from [19].

from the galactic plane. This goal was achieved by first selecting all the events received by the LAT in 12 years within a radius of 1 degree from the center of the Sculptor galaxy, and then applying the Li\&Ma method [22]. The probability that the triplet was due to a background fluctuation was found to be less than $8.3 \times 10^{-7}(5.3 \sigma)$. Then, to see if such a cluster of three events (called triplet) was somehow common for this ROI, the same data-set was used to build a histogram of the time intervals $\Delta t_{i}=t_{i+2}-t_{i}$ for each triplet formed by three consecutive events (Figure 5). The solid black line is the expected distribution using the Poisson statistics while the green and red distributions come from the actual data, respectively with and without the correction for the Bad Time Intervals. The orange vertical line corresponds to the $\Delta t$ observed for GRB 200415A and shows that in 12 years of observation (roughly 3 years of total livetime) only one triplet of photons had a smaller $\Delta t$ (but with a maximum TS $=16$ ). Finally, the probability of a chance temporal coincidence of this relatively rare triplet event with the GBM signal was calculated to be around 1 in million years.

A simple physical emission model was proposed to explain the production and detection of the $\mathrm{GeV}$ emission component, never observed before. It is known that a sudden crustal fracture of the magnetar can produce a powerful pulse of X-rays and soft gamma-rays, together with an ultra-relativistic outflow of plasma $(\Gamma \approx 100)[23,24]$. These travel in a practically empty space constantly swept by the steady wind from the magnetar, until they eventually reach a shell boundary called bow-shock. Here the light from the flare just passes through, while the ejected material interacts with the pile-up of interstellar gas. After the collision, the shock waves propagate inside the bow-shock shell and accelerate electrons to relativistic energies, which in the end produce GeV gamma-rays via synchrotron radiation in the shock-generated magnetic fields [19]. 


\section{Conclusion}

Magnetars are among the most extreme objects in the Universe and their rare form of emission known as giant flare may constitute a fraction of the current samples of short GRBs. Fermi-LAT and GBM Collaborations reported the high-energy detection—and the first at the $\mathrm{GeV}$ scale — of one of these events originated in the NGC 253 galaxy. Since a significant detection of pulsation was not clearly observed, the case about the magnetar origin remains still open. Nevertheless, putting all pieces together, the burst observed by GBM and LAT in April 2020 had all the features expected from an MGF: the burst light curve, its spectrum, the absence of GW in a nearby event, as well as the fact that it occurred in a star-forming galaxy. All these evidences are pointing to a magnetar origin for this event, with the unexpected discovery of a delayed very high-energy emission.

\section{References}

[1] Thompson, C. and Duncan, R. C. The soft gamma repeaters as very strongly magnetized neutron stars - I. Radiative mechanism for outbursts. Mon. Not. R. Astron. Soc. 275, 255-300 (1995).

[2] Frail, D. A., Kulkarni, S. R. and Bloom, J. S. An outburst of relativistic particles from the soft $\gamma$-ray repeater SGR1900+14. Nature 398, 127-129 (1999).

[3] Hurley, K. et al. An exceptionally bright flare from SGR 1806-20 and the origins of shortduration $\gamma$-ray bursts. Nature 434, 1098-1103 (2005).

[4] Hurley, K. et al. A giant periodic flare from the soft $\gamma$-ray repeater SGR1900+14. Nature 397, 41-43 (1999).

[5] Palmer, D. M. et al. A giant $\gamma$-ray flare from the magnetar SGR 1806-20. Nature 434, 1107 1109 (2005).

[6] Fenimore, E. E., Klebesadel, R. W. and Laros, J. G. The 1979 March 5 gamma-ray transient: was it a classic gamma-ray burst? Astrophys. J. 460, 964-975 (1996).

[7] Feroci, M. et al. A giant outburst from SGR 1900+14 observed with the BeppoSAX gamma-ray burst monitor. Astrophys. J. 515, L9-L12 (1999).

[8] Mazets, E. P. et al. A giant flare from a soft gamma repeater in the Andromeda galaxy (M31).

[9] Burns, E. et al. Identification of a Local Sample of Gamma-Ray Bursts Consistent with a Magnetar Giant Flare Origin. Astrophys. J. Lett. 907, L28 (2021).

[10] KAGRA collaboration. KAGRA: 2.5 generation interferometric gravitational wave detector. Nat Astron 3, 35-40 (2019).

[11] Perley, R. A., Chandler, C. J., Butler, B. J. and Wrobel, J. M. The Expanded Very Large Array: a new telescope for new science. Astrophys. J. 739, L1 (2011). 
[12] Roberts, O.J., Veres, P., Baring, M.G. et al. Rapid spectral variability of a giant flare from a magnetar in NGC 253. Nature 589, 207-210 (2021).

[13] Svinkin, D., Frederiks, D., Hurley, K. et al. A bright $\gamma$-ray flare interpreted as a giant magnetar flare in NGC 253. Nature 589, 211-213 (2021).

[14] Rekola, R. et al. Distance to NGC 253 based on the planetary nebula luminosity function. Mon. Not. R. Astron. Soc. 361, 330-336 (2005).

[15] Tohuvavohu, A. et al. Gamma-ray Urgent Archiver for Novel Opportunities (GUANO): Swift/BAT event data dumps on demand to enable sensitive sub-threshold GRB searches. Astrophys. J. 900, 35 (2020).

[16] Yang, J. et al. GRB 200415A: a short gamma-ray burst from a magnetar giant flare? Astrophys. J. 899, 106 (2020).

[17] Frederiks, D. et al. GRB 051103 and GRB 070201 as giant flares from SGRs in nearby galaxies. AIP Conf. Proc. 1000, 271-275 (2008).

[18] Ajello, M. et al. A decade of gamma-ray bursts observed by Fermi-LAT: the second GRB catalog. Astrophys. J. 878, 52 (2019).

[19] The Fermi-LAT Collaboration. High-energy emission from a magnetar giant flare in the Sculptor galaxy. Nat Astron 5, 385-391 (2021).

[20] VizieR Online Data Catalog NGC 2000.0 VII/118 (Sky Publishing, 1988).

[21] de Ruiter, H. R., Willis, A. G. and Arp, H. C. A Westerbork 1415 MHz survey of background radio sources. II. Optical identifications with deep IIIa-J plates. Astron. Astrophys. Suppl. 28, 211-293 (1977).

[22] Li, T. P. and Ma, Y. Q. Analysis methods for results in gamma-ray astronomy. Astrophys. J. 272, 317-324 (1983).

[23] Ioka, K., Razzaque, S., Kobayashi, S. and Mészáros, P. TeV-PeV neutrinos from giant flares of magnetars and the case of SGR 1806-20. Astrophys. J. 633, 1013-1017 (2005).

[24] Nakar, E., Piran, T. and Sari, R. Pure and loaded fireballs in soft gamma-ray repeater giant flares. Astrophys. J. 635, 516-521 (2005). 